\title{
CAN YEAST BE USED AS A SOURCE OF THE ANTINEURITIC VITAMIN IN INFANT FEEDING? *
}

\author{
AMY L. DANIELS, Ph.D. \\ Iowa Child Welfare Research Station, State University of Iowa \\ IOWA CITY
}

The reports of Eddy and Roper ${ }^{1}$ and Daniels and Byfield ${ }^{2}$ on the effect of adding substances containing the antineuritic vitamin ${ }^{3}$ to the diets of artificially fed infants suggests that at least part of the difficulty in feeding these babies is due to a too low concentration of the antineuritic vitamin in many of the feeding mixtures. Cow's milk has been shown to contain less of this vitamin than many other natural foods, ${ }^{4}$ the amount present being dependent on the vitamin content of the food of the cow, for it appears that "vitamins pass into the milk only as they are present in the diet of the mother." ${ }^{5}$ When cow's milk is diluted, a necessary procedure in feeding young. babies, unless some antineuritic vitamin is added from other sources, too little may be present in the feeding mixtures. The baby who is well and able to take his normal quota of food appears to get enough of this particular growth material to meet his needs. But the young baby or the baby with disturbed digestion who cannot take the usual amount of food may fail to gain because the antineuritic vitamin content of the food falls below his minimal requirement.

Eddy and Roper found that a specially prepared powder, made from the alcoholic extract of the pancreatic gland of lambs, when added to the diet of marasmic infants, stimulated growth. Previous experiments on rats had demonstrated that this powder contained the antineuritic vitamin. Daniels and Byfield, working with well babies, observed that many who were not gaining on food mixtures containing adequate amounts of the well known food constituents-protein, fat, carbohydrate and inorganic salts-increased in weight when the antineuritic vitamin (obtained from wheat embryo) was added to the feeding mixtures. In many instances these children were receiving food mixtures supplying from 115 to 130 calories on their theoretical weights-considerably more

\footnotetext{
* Received for publication, Aug. 18, 1921.

* This study was made possible through the courtesy of the Pediatric Department of the College of Medicine of the University of Iowa.

1. Eddy, W. H., and Roper, J. C.: Am. J. Dis. Child. 14:189 (Aug.) 1916

2. Daniels, A. L., and Byfield, A. H.: Am. J. Dis. Child. 18:546 (Dec.) 1919

3. The term antineuritic vitamin is used throughout the report to indicate the growth promoting vitamin designated as "Water Soluble B" by McCollum and his co-workers.

4. Osborne, T. B., and Mendel, L. B.: J. Biol. Chem. 34:573, 1918.

5. McCollum, E. T.; Simmonds, N., and Pitz, W.: J. Biol. Chem. 27:33, 1916.
} 
than is usually considered necessary. Growth in a number of instances continued until somewhat less than 100 calories per kilogram was being given. These observations suggest a possible explanation for the well known fact that the baby fed artificially needs more food than the breast fed baby.

In view of these findings it seems desirable to obtain some inexpensive, readily available source of this antineuric vitamin which may be added to the food of the very young infant or the baby who for one reason or another is unable to take a sufficient amount of food to ensure an adequate ingestion of the vitamin. Neither the wheat embryo extract used by Daniels and Byfield, nor the pancreas preparation of

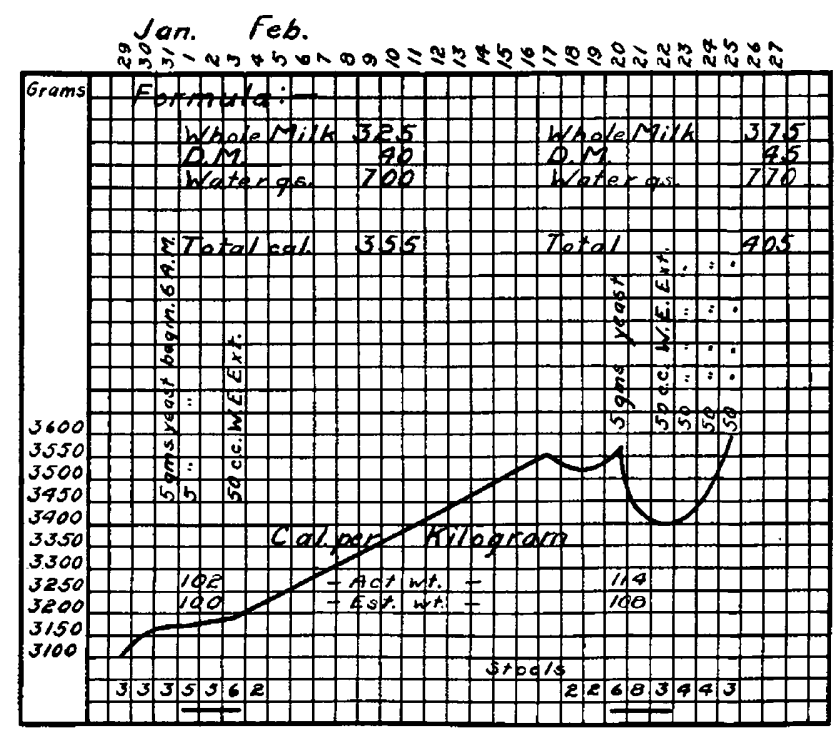

Fig. 1.-Sybil T.; horn Dec. 21; birth weight, $2.858 \mathrm{gm}$; aged, 11/4 months.

Eddy and Roper are easily available to the practitioner or the mother. The vegetable soup suggested ${ }^{6}$ by the former investigators as a source of the antineuritic vitamin has been used with much success for well babies over 4 months of age. It is, however, not well tolerated by the very young baby or the baby suffering from digestive disturbances. Both orange ${ }^{7}$ and tomato ${ }^{8}$ juice can be used as sources of the antineuritic vitamin, but considerably more must be used than is necessary to protect against scurvy; and the young baby or ill baby frequently cannot take enough to furnish the required amount.

6. Daniels, A. L., and Byfield, A. H.: loc. cit.

7. Byfield, A. H., and Daniels, A. L.: Am. J. Dis. Child. 19:349 (April) 1920. Osborne, T. B., and Mendel, L. B.: J. Biol. Chem. 42:465, 1920.

8. Osborne, T. B., and Mendel, L. B.: J. Biol. Chem. 41:451, 1920. 
In animal experimentation yeast ${ }^{9}$ has been used with much success as a means of furnishing this growth promoting vitamin, its potency in this respect being considerably greater than that of many foods tested, ${ }^{10}$ especially milk. ${ }^{11}$ Osborne and Mendel working with rats, observed that normal growth could be secured when $0.2 \mathrm{gm}$. per day was used as the only source of the vitamin, whereas 16 c.c. of milk was necessary to furnish an adequate amount. The possibility of using yeast as a means of increasing the antineuritic content of infants' food was suggested. The introduction of purin forming substances into the diets of infants did not commend itself, but since in all probability only a small amount of yeast would be needed to produce the desired results, it

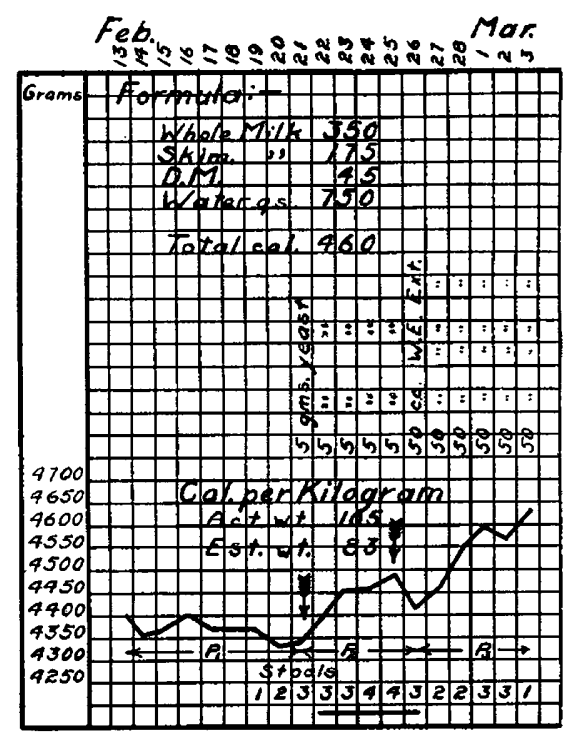

Fig. 2.-Clarice P.; born October 27; birth weight, 3,750 gm.; aged, 31/2 months

was reasoned that the good which might accrue would perhaps outweigh the theoretical objection. In the investigation from 2 to $10 \mathrm{gm}$. air dried pulverized yeast (Fleischmann's) were soaked in a small amount of water, subsequently boiled and added to the day's feeding. In certain instances, when no gain in weight resulted from the yeast additions, wheat embryo extract ( 50 c.c.) was substituted for the yeast, in order to make sure that the failure to gain was not due to a lack of the antineuritic vitamin. In some cases the wheat embryo extract was added for therapeutic purposes.

9. Osborne, T. B., and Mende1, L. B.: J. Biol. Chem. 31:149, 1917.

10. Osborne, T. B., and Mendel, L. B.: J. Biol. Chem. 32:309, 1917.

11. Osborne, T. B., and Mendel, L. B.: J. Biol. Chem. 34:537, 1918. 
In determining the influence on growth of a given vitamin addition to the diet of infants, several factors must be considered. The babies under observation should be as nearly normal as possible-that is, there should be no elevated temperatures, diarrhea, or other indications of indigestion or illness. The amount of food given must be sufficient to cover the physiologic requirements for age and weight. The feedings must be prepared with the greatest accuracy, as to amount of materials used and method of preparation. Care must be taken to see that all the food is taken and retained. It is most desirable that a stationary weight period of at least four days on a satisfactory food mixture should precede the period during which the added material is given. The

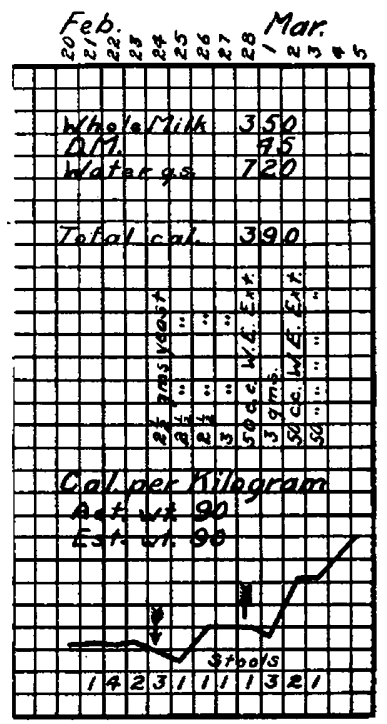

Fig. 3.-Keith M.; born December 27; birth weight, 3,595 gm.; age, 7 weeks.

character and number of the stools, both before and during the observation period, should be noted. If possible, the baby should be kept on the same formula, without the added vitamin, for four days following the period.

In testing the influence of yeast additions on growth, these methods, in so far as possible, were followed. The sixteen babies used for the observations, with the exception of two suffering from sudamina, were normal healthy babies from 5 weeks to 6 months of age. Details of the feeding mixtures, weights, etc., in a number of the more typical cases are given in the accompanying charts.

The most noticeable general effects of the yeast additions, especially with the younger babies, was the change in the number and character of the stools, a formed "safe" stool often becoming diarrheal. In many 
instances not only was the character of the stool changed, but the number per day was greatly increased even when comparatively small amounts of yeast were used. These frequent diarrheal stools were in a number of cases followed by sudden losses in weight. The results were sometimes so disastrous that it was necessary to institute corrective measures at once. For example, the addition of $5 \mathrm{gm}$. dried yeast (equivalent to about two-thirds of a fresh yeast cake) to the feedings of Sybil T., a 6 weeks old baby, was followed by frequent watery stools. After three feedings (twelve hours) it was necessary to change to an "eiweiss" milk formula to check the diarrhea. Three weeks later the yeast additions were again tested with results that were even more

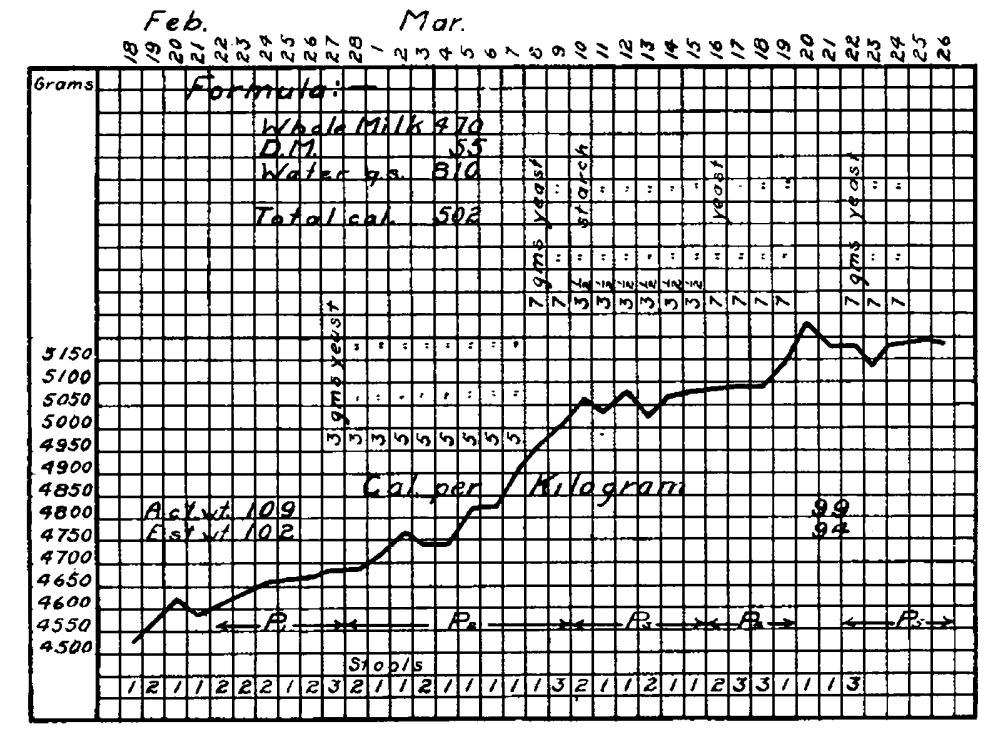

Fig. 4. - Gretchen S.; born Nov. 30, 1920; birth weight, 3,396 gm.; age, $2 \frac{1}{2}$ months.

marked than during the first yeast period. At this time the feedings were not well retained, and the stools increased from two per day to six and eight, respectively. There was a sudden loss in weight, and after a twenty-four hour trial the baby was returned to its previous formula to which were added 50 c.c. of our wheat embryo extract.

The addition of 5, 7 and $21 / 2 \mathrm{gm}$. yeast to Robert L.'s feedings produced somewhat similar results-marked diarrhea and loss in weight when the larger amounts of yeast were used. With the smaller amount of yeast-21/2 $\mathrm{gm}$.- - the stools were less frequent and formed, but apparently not enough of the antineuritic vitamin had been added with this small amount of yeast to produce any apparent physiologic effect; the subsequent addition of 50 c.c. of wheat embryo extract resulted in 
an increase in weight. It would seem that in this case any good effects that might have accrued from the antineuritic content of the larger amounts of yeast were counteracted by the purgation produced.

The amount of the antineuritic vitamin necessary to stimulate growth in any given case depends obviously on the amount contained in the feeding mixture. In certain instances, it may be possible to use yeast with no untoward results, provided the antineuritic vitamin content of the food is only slightly below the minimum requirement, and, therefore, only a small additional amount is necessary. In the case of Robert L. and Keith M., 21/2 gm. was not sufficient to produce any noticeable effects on growth, whereas the addition of $21 / 2 \mathrm{gm}$. to the

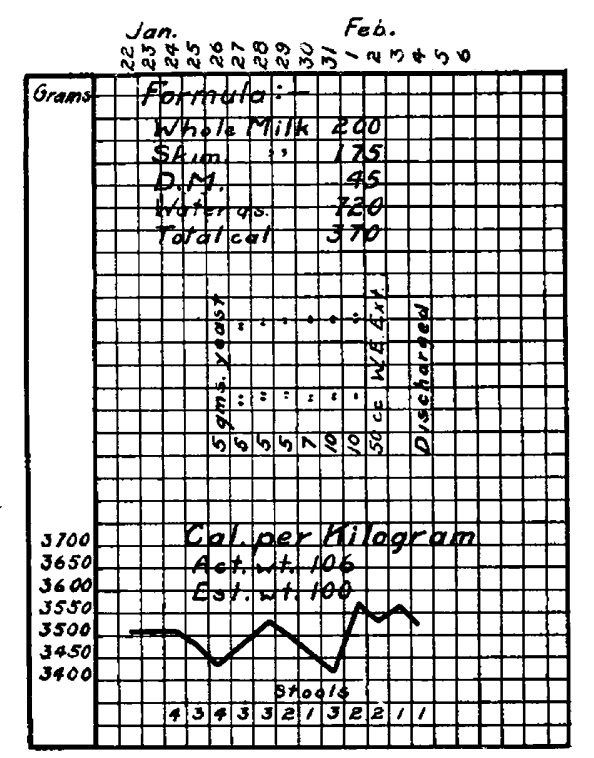

Fig. 5.-Walter A.; born Dec. 12, 1920; birth weight, 3,262 gm.; age, 6 weeks.

diet of Leona L. caused a slight gain in weight- $60 \mathrm{gm}$. in five daysin spite of frequent stools. The substitution of 50 c.c. of wheat embryo extract, however, resulted in an increase of $190 \mathrm{gm}$. in five days. Similar increases were produced by the addition of wheat embryo extract in the other cases where yeast had been tested. In view of the marked effect of the yeast on peristalsis, in none of these children could larger amounts of yeast have been used.

With babies suffering from constipation, yeast, possibly, may be used with some degree of success, although we believe that there are better methods of meeting this problem. In tour of our cases constipated stools became normal in consistency during the yeast additions. In one case (Gretchen S.) not only was the constipation relieved but 
there was a considerable increase in weight. The addition of 3.5 and $7 \mathrm{gm}$. yeast, respectively, was followed by a weight increase of 370 $\mathrm{gm}$. in eleven days. When yeast was omitted and starch $(3 \mathrm{gm}$.) equivalent to the amount carried in the $7 \mathrm{gm}$. yeast was substituted, the weight became stationary, indicating that the gain was due to the antineuritic vitamin of the yeast rather than to the starch contained therein. A subsequent addition of yeast produced a slight gain in weight- 50 grams in 5 days. At this time the low caloric value of the food-94 grams per kilogram, in all probability accounts for the small gain during the second yeast period.

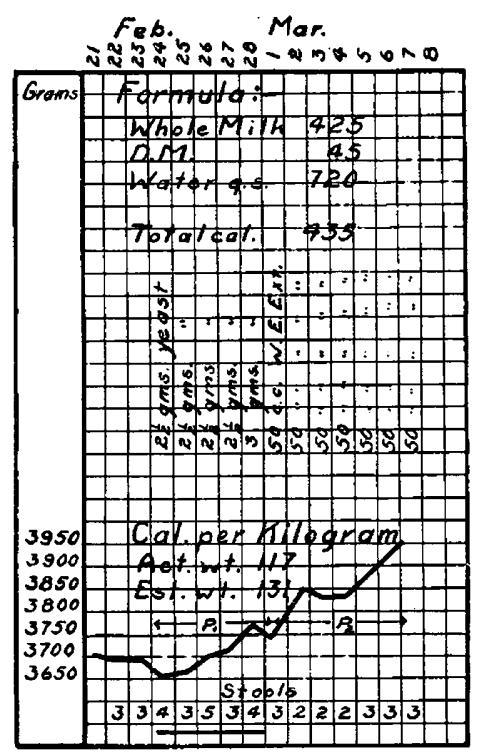

Fig. 6.-Leona L.; born January 8; birth weight, 3,040 gm.; age, 6 weeks.

At the time our other studies were being made there were in the hospital two rather severe cases of sudamina. Since Hawk ${ }^{12}$ and his co-workers had obtained such good results with Baker's yeast in furunculosis, acne and other skin infections, it seemed desirable to test the effects of yeast on these two babies. In one case the infection had been running for about ten days, in the other case a somewhat shorter time. With both babies fairly large amounts of yeast-from 5 to $10 \mathrm{gm}$.were used. During the period our staff were questioned each morning with regard to the condition of the babies. For a time several of them did not know that any treatment was being tried, so that their opinion

12. Hawk, P. B.; Knowles, F. C.; Rehfus, M. E., and Clarke, I A.: J. A. M. A. 69:1243, 1917. 
was quite unbiased. There appeared to be no untoward results during the yeast additions, nor was there any apparent beneficial effects on the course of the infection. After days of yeast therapy the boils persisted. Indeed, in one other case (R.L.) boils developed during yeast feeding. The boils finally yielded in three days to the application of a dilute solution of iodin and benzol. In the cases noted there was no increase in weight, although fairly large amounts of yeast were used; nor was any diarrhea produced. The subsequent addition of 50 c.c. wheat embryo extract to the feedings was also without effect, indicating that stationary

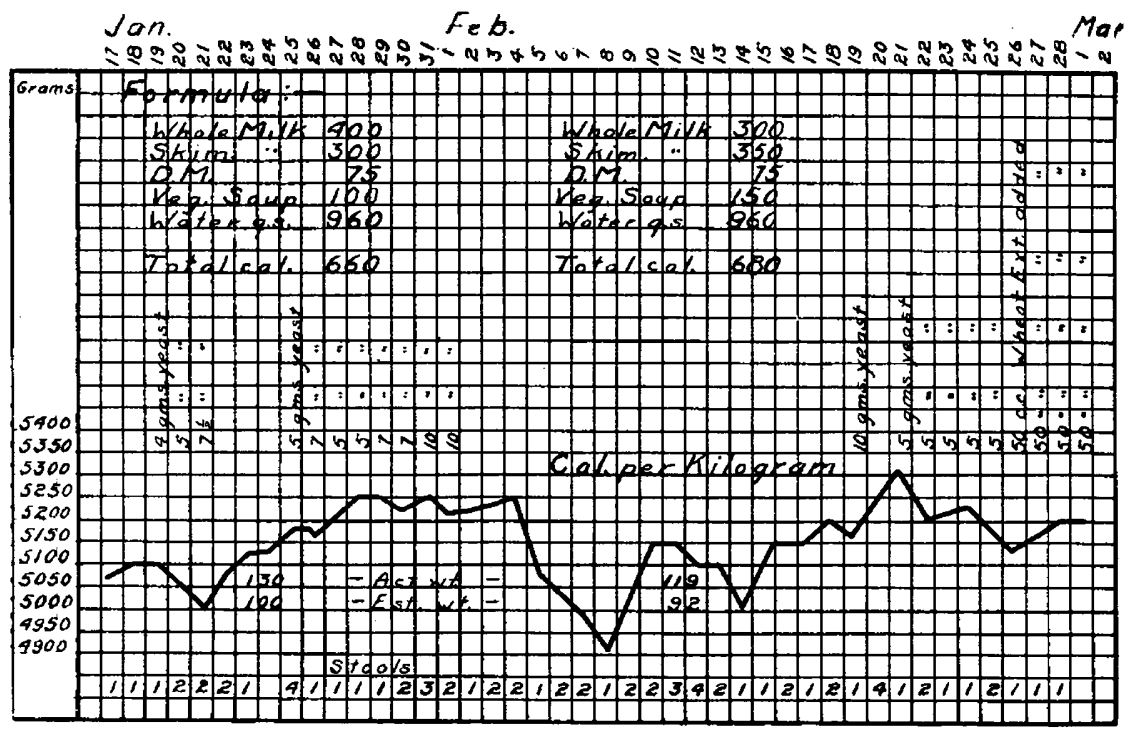

Fig. 7.-Robert G.; born July 10, 1920; birth weight, 3,247 gm.; age, 6 months.

weight in these cases was not due to a lack of the antineuritic vitamin. A failure to gain during an infection of this kind has been observed frequently.

Altogether, our experience with yeast points to the conclusion that it should not be used as a means of increasing the antineuritic content of infants' foods. All the babies under observation were well babiesthat is, well from the standpoint of digestion. The addition of yeast, even in small amounts, resulted in diarrhea in a large number of cases, the untoward effects with the younger babies being more marked than with the older ones. Since in all probability the artificially fed baby most in need of some additional antineuritic vitamin will be the young baby, or the baby suffering from disturbed digestion, it is obvious that some substance other than yeast must be found as a means of supplying this. 
A recent paper by Ladd ${ }^{13}$ on the effects of yeast in infant feeding, published since the above report was written, corroborates our findings in certain respects. The details of the investigation are not given, therefore, critical comparisons cannot be made. Ladd, however, was unable to obtain evidence that yeast had any therapeutic effect on the course of furunculosis in infants; nor was there any evidence in his studies that yeast in the amounts given influenced the rate of growth. In one case fermentative diarrhea developed soon after the yeast was begun.

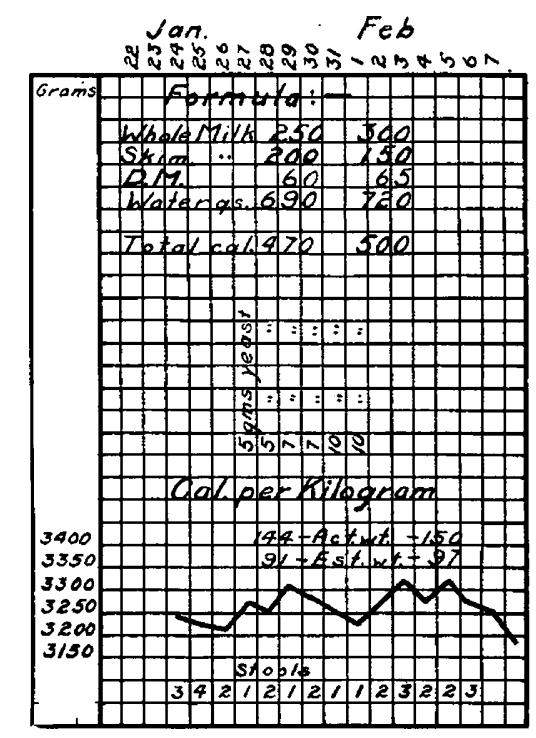

Fig. 8.-Wilbur Van H.; born Oct. 5, 1920; birth weight, 3,170 gm.; age, $3 \frac{1 / 2}{}$ months.

\section{REPORT OF CASES}

CASE 1.-Clarice $P$. The stimulation of growth during Period 2 was apparently due to the yeast additions, since it followed a period of stationary weight. During the yeast period the stools became watery and more frequent. The substitution of 50 c.c. of wheat embryo extract (Period 3) resulted in a gain of $230 \mathrm{gm}$. in five days, against an increase of $150 \mathrm{gm}$. in the five day yeast period.

CASE 2.-Robert L. The addition of 5 and $7 \mathrm{gm}$. yeast, respectively, to the diet of Robert L., a baby who had always been a difficult feeding case, caused an increase in the number of stools and a subsequent loss in weight. Two and one-half grams were without effect on growth. The addition of 50 c.c. of wheat embryo extract resulted in an increase in weight.

CASE 3.-Walter A. Yeast produced no untoward results nor did it stimulate growth. Apparently, his failure to gain was due to some cause other than a lack of the antineuritic vitamin.

CASE 4.-Julia $M c C$. The addition of $3 \mathrm{gm}$. yeast resulted in a temporary gain in weight. The increase in the number of diarrheal stools made it necessary to discontinue the yeast.

13. Ladd, M.: Arch. Pediat. 38:423 (July) 1921. 
CASE 5.-Leona $L$. The addition of $2.5 \mathrm{gm}$. yeast produced a slight gain in weight (Period 1). This, however, was accompanied by an increase in the number of stools. The substitution of 50 c.c. wheat embryo extract (Period 2) was more effective than the yeast. During this period the stools became normal.

CASE 6. - Walter $\operatorname{Van} H$. was suffering from a severe case of sudamina. The addition of yeast in fairly large amounts- $-5,7$ and $10 \mathrm{gm}$. , respectivelywas without effect on growth; nor did the yeast appear to affect the course of the infection, which was subsequently cleared up after three days' treatment with a dilute solution of iodin and benzol.

CASE 7.-Mary B. The addition of $5 \mathrm{gm}$. yeast (dry weight) to the diet of this 7 weeks old baby produced diarrhea, manifested by many loose, watery stools.

CASE 8.-Sybil $T$. The addition of $5 \mathrm{gm}$. yeast produced severe diarrhea in a baby, 6 weeks old. Similar results were obtained three weeks later. At both times the effects were so marked that it was necessary to discontinue the yeast feedings.

CASE 9.-Gretchen $S$. The addition of yeast to the diet apparently stimulated growth without producing the untoward results observed in many of our other babies. This baby, however, was always quite constipated. The yeast additions were followed by soft yellow stools. During Period 3 the effect of $3.5 \mathrm{gm}$. of arrow starch (the amount contained in the yeast used) was tested. The weight remained stationary, indicating that the gain in Periods 2 and 4 were the results of the yeast additions. During Period 5 the caloric value of the food ( 94 calories per kilo on her theoretical weight) was too low to admit of gain in weight.

CASE 10.-Keith $M$. The addition of 2.5 and $3 \mathrm{gm}$., respectively, of dry yeast to the milk feedings produced no gain in weight. The effect on the stool was not marked. The substitution of 50 c.c. wheat embryo extract for the yeast resulted in a subsequent gain in weight.

CASE 11.-Robert $G$. The addition of yeast to the diet was without influence on growth. It also appeared to have no effect on a marked condition of sudamina. This baby apparently was not "well born" and had been a difficult feeding case from the first. The failure to grow was not caused by a lack of the antineuritic vitamin, as shown by the fact that the addition of 50 c.c. of our wheat embryo extract was also without effect. 